'Departamento de Nefrología, Escuela de Medicina, Facultad de Medicina, Pontificia Universidad Católica de Chile. Santiago, Chile. ${ }^{2}$ Departamento de Laboratorios Clínicos, Escuela de Medicina, Facultad de Medicina, Pontificia Universidad Católica de Chile. Santiago, Chile.

Fuentes de financiamiento: No hubo.

Los autores declaran no tener conflictos de interés

Recibido el 21 de agosto de 2017, aceptado el 12 de noviembre de 2017

Correspondencia a: Dr. Juan Pablo Huidobro Diagonal Paraguay $3624^{\circ}$ piso, Santiago, Chile. jpablohuidobro@gmail.com

\section{Creatinina y su uso para la estimación de la velocidad de filtración glomerular}

\author{
JUAN PABLO HUIDOBRO E. ${ }^{1}$, RODRIGO TAGLE ${ }^{1}$, \\ ANA MARÍA GUZMÁN ${ }^{2}$
}

\section{Estimation of glomerular filtration rate with creatinine}

\begin{abstract}
Glomerular filtration rate (GFR) is the best approximation to global renal function and its estimation is of great relevance for clinical practice. Since the measurement of GFR by reference methods is complex, costly and not widely available, its routine evaluation is performed using endogenous biomarkers. Within these, creatinine is the most commonly used. It allows the estimation of GFR by means of its clearance or by formulas based on its concentration on plasma. Creatinine measurement should be performed using enzimatic methods as they confer more accurate values than Jaffe methods, especially for normal and low creatinine levels.
\end{abstract}

(Rev Med Chile 2018; 146: 344-350)

Key words: Creatinine; Glomerular Filtration Rate; Kidney Function Tests.
$\mathrm{E}$ xiste consenso en que la velocidad de filtración glomerular (VFG) es la mejor aproximación a la función renal global ${ }^{1}$. Tener una estimación de la función renal es de gran relevancia en la práctica clínica, pues permite la detección y etapificación de la enfermedad renal crónica $(E R C)^{2}$, la dosificación de medicamentos, definir la posibilidad de ciertos exámenes radiológicos, la determinación del potencial de donación renal y la decisión de inicio de terapia de reemplazo renal.

Para acercarnos al valor de la VFG podemos medir el aclaramiento o clearance de una sustancia exógena o estimar el de una sustancia endógena, entendiendo el concepto de clearance como el volumen de plasma en que una sustancia es depurada por unidad de tiempo ${ }^{3}$. El clearance de una sustancia ideal, esto es, que se filtre libremente a nivel glomerular, no se reabsorba ni se secrete a nivel tubular, es lo que se entiende como medición de la VFG (mVFG). Esto se puede realizar con inulina, un polímero de fructosa que es considerado el estándar de oro para estos efectos, o mediante otros marcadores como el iotalamato o el iohexol, los que también son considerados métodos de referencia ${ }^{4}$. Dado que la $\mathrm{mVFG}$ requiere la administración endovenosa de una sustancia exógena y la medición seriada de su concentración en plasma y/u orina, en la práctica clínica habitual utilizamos biomarcadores endógenos que permiten una estimación de la VFG. Ejemplos de biomarcadores cuyas concentraciones están determinadas por la VFG son la creatinina, cistatina C, beta 2 microglobulina y proteína beta trace (BTP $)^{5,6}$. De éstos, el más utilizado es la creatinina, la que puede ser utilizada para calcular un clearance que estime la VFG. A partir de la creatinina, a su vez, se han generado ecuaciones que permiten una estimación de la VFG combinando el valor sérico de creatinina con otras variables dependientes del paciente (edad, peso, raza, entre otras).

En esta revisión ahondaremos en las características de la creatinina como biomarcador, en sus métodos de medición y sus respectivos rendimientos analítico y clínico. Finalmente, expondremos las fórmulas más utilizadas para la estimación de la VFG basadas en creatinina con énfasis en sus fortalezas y debilidades. 


\section{La creatinina como biomarcador}

La creatinina es un producto metabólico no enzimático de la creatina y la fosfocreatina, que en condiciones normales se produce a una tasa constante desde el tejido muscular esquelético (alrededor de $2 \%$ por día de la reserva total de creatina) (Figura 1). Es una molécula pequeña (113 Dalton) y no circula unida a proteínas plasmáticas, por lo que se filtra libremente a nivel glomerular ${ }^{7}$. No se reabsorbe, pero se secreta por el túbulo proximal en un porcentaje variable, que aumenta a medida que progresa la insuficiencia renal, lo que determina que el clearance de creatinina sobreestime el valor real de la VFG y que esta situación aumente a medida que progresa la falla renal. En condiciones normales la excreción extrarrenal de creatinina es mínima; sin embargo, a medida que decae la VFG se produce un aumento de su excreción intestinal, favorecida por proliferación de flora intestinal capaz de degradarla ${ }^{8}$.

Otra limitación de la creatinina como biomarcador es que está determinada por factores independientes de la VFG, como son la masa muscular, la ingesta proteica, el ejercicio y el uso de medicamentos que pueden bloquear la secreción tubular, como cimetidina y trimetoprim ${ }^{9}$. Además, los fibratos podrían aumentar la producción de creatinina a nivel muscular ${ }^{10}$ (Tabla 1). Finalmente, se debe considerar que la relación de la creatinina con la VFG no es lineal, sino que exponencial, lo que puede llevar a subestimar la importancia de pequeñas variaciones a niveles bajos de creatinina y sobreestimar la importancia de variaciones mayores a niveles más altos (Figura 2).

\section{Medición de la creatinina}

En 1886, Max Jaffe describió la reacción de la creatinina con el ácido pícrico en medio alcalino. Esta reacción generaba un aducto de color rojizo, cuya tasa de formación era proporcional a la cantidad de creatinina en la muestra ${ }^{11}$. Desde su descripción se sabía que otros elementos presentes en el plasma también producían la reacción, entre-

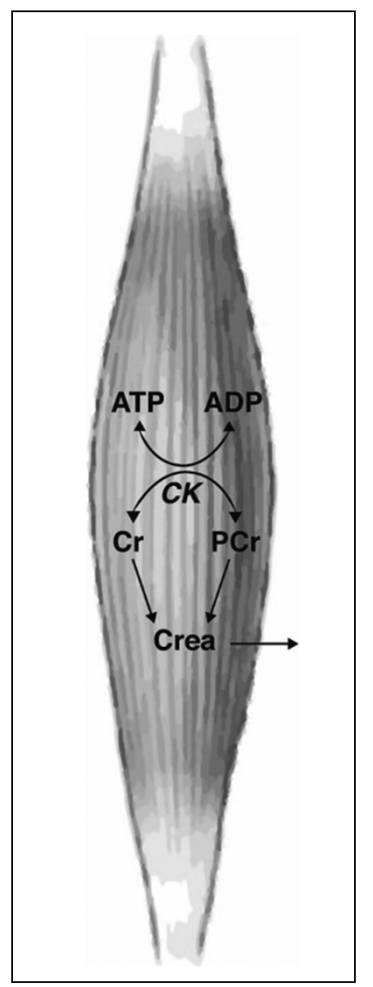

Figura 1. ATP: Adenosin trifosfato; ADP: Adenosin difosfato; CK: Creatinquinasa; Cr: Creatina; PCr: Fosfocreatina; Crea: Creatinina.

Tabla 1. Factores que alteran el valor de creatinina sérica independiente de la velocidad de filtración glomerular

\begin{tabular}{|ll|}
\hline Factor & Mecanismo y efecto \\
\hline Masa muscular & Gran masa muscular eleva los niveles séricos mientras que sarcopenia los disminuye \\
\hline Ingesta proteica & $\begin{array}{l}\text { Dietas ricas en proteínas, suplementos proteicos o de creatinina aumentan niveles } \\
\text { séricos de creatinina; dietas vegetarianas los disminuyen }\end{array}$ \\
\hline Ejercicio & $\begin{array}{l}\text { Ejercicio extenuante puede producir rabdomiolisis, elevando los niveles séricos de } \\
\text { creatinina }\end{array}$ \\
\hline Drogas (cimetidina, trimetoprim) & $\begin{array}{l}\text { Bloquean la secreción tubular de creatinina, elevando sus niveles séricos } \\
\text { Fibratos }\end{array}$ \\
\hline
\end{tabular}




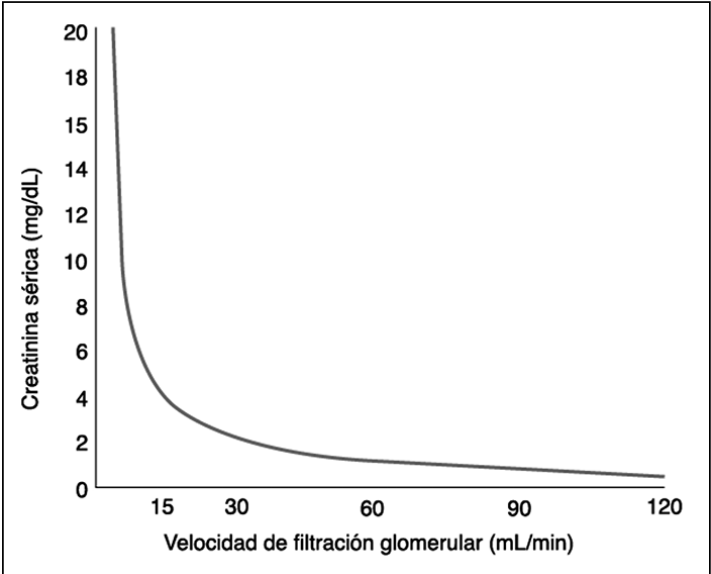

Figura 2.

gando un valor de medición de la creatinina más elevado que el real; estos elementos se denominaron interferentes positivos o pseudocromógenos y corresponden principalmente a las cetonas, glucosa y proteínas. Además, se sabía que existían elementos que interferían directamente con la medición del color formado, entregando un valor de medición de la creatinina menor al real; estos elementos se denominaron interferentes negativos y corresponden principalmente a la bilirrubina. Para corregir estos problemas inherentes a la técnica de Jaffe original, se han realizado ajustes a lo largo del tiempo. En primer lugar se cambió la medición del color total (método colorimétrico de punto final) a un método cinético, en que se medía la formación del color en un tiempo determinado, con lo cual se minimizaba la contribución de los pseudocromógenos, los que reaccionaban con el ácido pícrico más tardíamente. Además, se minimizó la interferencia de los interferentes negativos (bilirrubina) con la determinación de un blanco. Posteriormente, se decidió incorporar un valor fijo de compensación para el efecto de los pseudocromógenos que no era corregido por la medición cinética. Esto es lo que se conoce como método colorimétrico cinético compensado o Jaffe compensado ${ }^{12}$.

Pese a todos los intentos por mejorar los métodos colorimétricos, su rendimiento a niveles normales o bajos de creatinina es subóptimo al comparar con un método referencia (espectrometría de masa por dilución isotópica o IDMS $)^{13}$, por lo que surgió la necesidad de medir la creatinina por un método con mejor rendimiento. De esta manera, en 1986, cien años después de la publicación del trabajo de Jaffe, se publicó el método enzimático de medición de creatinina ${ }^{14}$. Éste se basa en someter a la creatinina a varios procesos enzimáticos que terminan con la formación de peróxido de hidrógeno, el que por una reacción con peroxidasa forma un color rojo intenso cuya máxima absorbancia ocurre a una longitud de onda de $510 \mathrm{~nm}$. La formación de color es proporcional a la cantidad de creatinina de la muestra. Los métodos enzimáticos no se ven afectados por la presencia de pseudocromógenos, por lo que son más específicos para la medición de creatinina, pero sí pueden verse afectados por concentraciones altas de bilirrubina, que puede actuar como interferente negativo ${ }^{15}$.

En 2006, la National Kidney Disease Education Program (NKDEP) publicó las recomendaciones analíticas para la medición de creatinina. En ellas se estableció que los métodos de medición deben tener un sesgo (diferencia del valor medido contra el real) menor a 5,1\%, una imprecisión (variabilidad) menor a $3,2 \%$, un error total deseable menor a 7,6\% y máximo aceptable de $11,4 \%$. Estos requerimientos deben traducirse en que el error en el cálculo de la VFG aportado por la medición no debe superar $10 \%{ }^{16}$.

Para evaluar el rendimiento de los diferentes métodos enzimáticos disponibles en el mercado, en 2011 se realizó un estudio multicéntrico en 25 laboratorios de Francia, en los que compararon 12 métodos enzimáticos para 5 muestras de suero con diferentes concentraciones de creatinina medidas por IDMS. Para concentraciones iguales o mayores a $0,84 \mathrm{mg} / \mathrm{dL}$, todos los métodos tuvieron valores de sesgo e imprecisión que no superaron los límites establecidos por la NKFEP. Para un valor de $0,4 \mathrm{mg} / \mathrm{dL}$, la variabilidad del sesgo e imprecisión de los diferentes ensayos fue mayor, pero la mayoría cumplía con los requisitos de un error total menor a $11,4 \%{ }^{17}$. Para comparar con métodos de Jaffe, un trabajo publicado en 2013 demostró que, para concentraciones de 0,4, 0,84 y $1,1 \mathrm{mg} / \mathrm{dL}, 3$ métodos de Jaffe compensados no cumplían con los requerimientos de sesgo menor a 5,1\% ni de error total deseable para ninguna de las muestras, mientras que los ensayos por método enzimático sí lo hacían ${ }^{18}$.

Las implicancias del error aportado por el método de Jaffe son relevantes en la clínica. Para 
valores de creatinina entre 0,4 y $1,1 \mathrm{mg} / \mathrm{dL}$, la medición por Jaffe compensado presenta una sobreestimación de la creatinina mayor a $10 \%$ y que puede llegar hasta $30 \%$. Esto se traduce en que al utilizar estos valores de creatinina para calcular una VFG, 36,7\% de los pacientes son clasificados con ERC etapa 3 siendo que su VFG calculada con una creatinina obtenida por método de referencia era mayor a $60 \mathrm{ml} / \mathrm{min} / 1,73 \mathrm{~m}^{2,19}$. Para pacientes con VFG entre $60-90$ y $90-120 \mathrm{ml} / \mathrm{min} / 1,73 \mathrm{~m}^{2} \mathrm{el}$ cálculo de VFG mediante fórmulas de Chronic Kidney Disease Epidemiology Collaboration (CKD-EPI) y Modification of diet in renal disease (MDRD) utilizando un valor de creatinina obtenida por Jaffe compensado, presentaba un error mayor a $10 \%$ entre 21,6 y $40,6 \%$ de los pacientes, mientras que para ensayos realizados con creatinina enzimática el porcentaje de pacientes con error en el cálculo de la VFG mayor a $10 \%$ era menor a $10 \%{ }^{20}$.

Existe, por tanto, evidencia concluyente de que los métodos enzimáticos son superiores a los métodos colorimétricos por Jaffe compensado, sobre todo para valores normales o bajos de creatinina, en los que el error aportado por los ensayos basados en el principio de Jaffe podría llevarnos a clasificar a pacientes sanos incorrectamente en el grupo de pacientes con insuficiencia renal ${ }^{21}$. Desde el punto de vista clínico, es aconsejable que el seguimiento de los pacientes con creatinina considere el método de medición al momento de interpretar los resultados.

\section{Clearance de creatinina}

La creatinina es el biomarcador endógeno más utilizado para la estimación de la VFG. El cálculo de su aclaramiento se fundamenta en que es filtrada libremente y no se reabsorbe a nivel tubular, por lo que la cantidad absoluta excretada debiera ser constante entre individuos con la misma producción de creatinina y función renal estable ${ }^{8}$. La relación entre la cantidad de creatinina excretada (volumen urinario por concentración de creatinina en la orina) y la concentración de creatinina plasmática es el volumen que fue aclarado. Este valor debe dividirse por unidad de tiempo para calcular una tasa de aclaramiento. Normalmente se realiza en $24 \mathrm{~h}$, por lo que el valor resultante debe ser dividido por el número de minutos de un día, pero podría realizarse en tiempos más acotados sin un efecto significativo en su valor ${ }^{22}$.

Sus principales limitaciones radican en que, como hemos señalado, la creatinina se secreta en el túbulo proximal, por lo que su clearance sobreestima la VFG real. Además, la recolección de orina es engorrosa y susceptible de errores, los que dificultan la interpretación de los datos obtenidos.

\section{Fórmulas de estimación de la VFG basadas en creatinina}

Existen muchas fórmulas para la estimación de la VFG que se basan en creatinina. De ellas, analizaremos las 3 más ampliamente utilizadas (Cockroft-Gault, MDRD y CKD-EPI) y una recientemente publicada (FAS).

\section{Cockroft-Gault}

Se formuló con las creatininurias de $24 \mathrm{~h}$ de 249 hombres entre 18 y 92 años y se validó contra el clearance de creatinina de 236 pacientes, de los cuales sobre $90 \%$ eran hombres ${ }^{23}$. Con esto se obtuvo una fórmula que fue superior a las Jelliffe ${ }^{24} \mathrm{y}$ Edwards-Whyte ${ }^{25}$, utilizadas hasta ese momento.

Es importante destacar que esta fórmula estima el clearance de creatinina y no la VFG, lo que en sí es un defecto, ya que, como hemos analizado anteriormente, el clearance de creatinina sobreestima la VFG real. Otras limitaciones de este estudio son que fue realizado en tiempos en que los ensayos para medición de creatinina no eran estandarizados, se basa en el peso como representante de la masa muscular (por lo que la validez externa estaría limitada en amputados, pacientes con distrofias musculares y en poblaciones en que el peso pudiera estar influido en un mayor porcentaje por la masa grasa) y establece arbitrariamente que la masa muscular de las mujeres es $85 \%$ de la de los hombres, lo que no necesariamente es cierto.

Pese a todo lo anterior, gran parte de los estudios farmacológicos están realizados con esta fórmula, por lo que aún es utilizada para ajuste de fármacos. Además, su simpleza matemática y su gran difusión la han perpetuado en la práctica clínica.

\section{MDRD}

Se desarrolló en 1999 en 1.628 pacientes del estudio de modificación de la dieta en enfermedad 
renal $^{26}$. Se crearon dos fórmulas de estimación de la VFG a partir de datos de creatinina y otras variables para comparar con VFG medida por clearance de ${ }^{125}$ I-iotalamato en 1.070 pacientes con enfermedad renal. Estas fórmulas incluyeron otras variables como nitrógeno ureico, albúmina, género y raza, y luego se validaron en una muestra de 558 pacientes de la misma base de datos. Tanto la fórmula de 4 variables (MDRD-4) como la de 6 (MDRD-6) tuvieron mejor rendimiento que la fórmula de Cockroft-Gault en relación al estándar de oro.

En 2006 esta fórmula fue corregida para métodos trazables de medición de la creatinina (estandarizados contra método de referencia) ${ }^{27}$.

Algunas limitaciones de esta fórmula es que fue desarrollada exclusivamente en pacientes con enfermedad renal, por lo que su rendimiento en pacientes con VFG normal es menor. Además, excluyó en su fase de formulación a menores de 18 y mayores de 70 años, diabéticos tipo 1, diabéticos insulino requirentes, embarazadas y trasplantados. Si bien ha sido validada en algunos de estos grupos $^{28}$, su principal limitación parece estar dada para la estimación de la VFG en pacientes con función renal normal.

\section{CKD-EPI}

En 2009 se publicó la fórmula del grupo colaborativo CKD-EPI, formado por varios de los autores de la fórmula MDRD ${ }^{29}$. Esta fórmula se creó en base a los datos de 8.254 pacientes de 10 estudios y se validó en 3.896 pacientes de otros 16 estudios. Todas las creatininas fueron medidas por método enzimático en un solo laboratorio y se incluyó pacientes con y sin enfermedad renal (VFG promedio fue de $88 \mathrm{ml} / \mathrm{min} / 1,73 \mathrm{~m}^{2}$ ). La fórmula creada se comparó con clearance de iotalamato y mostró mejor rendimiento que MDRD para valores de VFG mayor a $60 \mathrm{ml} / \mathrm{min} / 1,73 \mathrm{~m}^{2}$ y similar rendimiento para los pacientes con VFG menor a $60 \mathrm{ml} / \mathrm{min} / 1,73 \mathrm{~m}^{2}$.

Pese a que desde su publicación ha sido considerada la mejor manera de estimar la VFG, la fórmula CKD-EPI tiene algunas limitaciones: para poder aplicarse se debe contar con un método trazable de medición de creatinina. Además, $87 \%$ de los pacientes en el grupo de formulación eran menores de 65 años, por lo que su rendimiento en población adulta mayor pudiese ser limitado.

\section{FAS}

Esta fórmula nació como un intento de poder utilizar una sola ecuación para los distintos grupos etarios (FAS: full age spectrum) ${ }^{30}$, considerando que para pacientes pediátricos la fórmula más utilizada es la de Schwartz, para pacientes jóvenes $y$ adultos CKD-EPI y para adultos mayores las recientemente creadas fórmulas de Berlín (BIS-1 y BIS-2 $)^{31}$. Se creó una ecuación basada en la fórmula de Pottel, que permite estimar la VFG en niños y se amplió su aplicabilidad a diferentes grupos etarios al incorporar el concepto de creatinina normalizada; esto es, la relación de la creatinina sérica y el valor promedio de creatinina para ese grupo etario. Esta fórmula se validó posteriormente en 6.870 individuos de diferentes estudios que tenían medición de la VFG (ya fuera por clearance de inulina, iotalamato o iohexol). Comparada con CKD-EPI, FAS fue superior en la clasificación de adultos entre 18 y 70 años con VFG entre 60-90 $\mathrm{ml} / \mathrm{min} / 1,73 \mathrm{~m}^{2}$ y comparable en el grupo con VFG entre $30-60 \mathrm{ml} / \mathrm{min} / 1,73 \mathrm{~m}^{2}$, pero inferior en los que tenían una VFG menor a 30 o mayor a $90 \mathrm{ml} / \mathrm{min} / 1,73 \mathrm{~m}^{2}$. En adultos mayores, FAS fue superior a CKD-EPI en el grupo con VFG $<60$ $\mathrm{ml} / \mathrm{min} / 1,73 \mathrm{~m}^{2}$ mientras que CKD-EPI mostró mejor rendimiento en la clasificación de adultos mayores con VFG mayor a $60 \mathrm{ml} / \mathrm{min} / 1,73 \mathrm{~m}^{2}$.

Limitaciones de este estudio es que los valores promedio de creatinina para cada grupo etario fueron obtenidos de un estudio en población caucásica. Además, los métodos de medición de VFG fueron diferentes en las distintas cohortes de validación.

\section{Conclusiones}

La estimación de la función renal de manera confiable es una necesidad en la práctica clínica, lo que ha impulsado esfuerzos a lo largo de la historia por encontrar mejores biomarcadores y fórmulas de estimación de la VFG. La creatinina se ha consolidado en la historia como el biomarcador preferido para estimar la VFG y su medición se ha ido perfeccionando con el tiempo, llevando a cuantificaciones progresivamente más exactas. El uso de métodos enzimáticos de medición de la creatinina permite obtener un valor más real que con métodos de Jaffe, sobre todo en individuos con función renal normal. El cambio de medición 
desde métodos colorimétricos por Jaffe a ensayos enzimáticos se ha visto limitado fundamentalmente por motivos económicos.

Finalmente, las distintas fórmulas de estimación de la VFG tienen limitaciones que impiden su aplicabilidad universal, por lo que, por el momento, la estimación de la VFG se mantiene como un desafío que debe ser individualizado para cada paciente.

Agradecimientos: Los autores agradecen a TM Edinson López Herrera, por su interés y trabajo en el perfeccionamiento de los métodos de medición de creatinina.

\section{Referencias}

1. Lesley A, Stevens MD, Josef Coresh MD Ph.D. Tom Greene PD, Andrew S. Levey MD. Assessing Kidney Function - Measured and Estimated Glomerular Filtration Rate. N Engl J Med 2006; 2473-83.

2. Levey AS, Coresh J, Balk E, Kausz AT, Levin A, Steffes MW, et al. National Kidney Foundation practice guidelines for chronic kidney disease: evaluation, classification, and stratification. Ann Intern Med 2003; 139 (2): 137-47.

3. Swan SK. The search continues-an ideal marker of GFR. Clin Chem 1997; 43: 913-4.

4. Vart P, Grams ME. Measuring and Assessing Kidney Function. Semin Nephrol 2016; 36 (4): 262-72.

5. Dharnidharka VR, Kwon C, Stevens G. Serum cystatin $\mathrm{C}$ is superior to serum creatinine as a marker of kidney function: A meta-analysis. Am J Kidney Dis 2002; 40 (2): 221-6.

6. White CA, Ghazan-Shahi S, Adams MA. Trace protein: a marker of GFR and other biological pathways. Am J Kidney Dis 2015; 65 (1): 131-46.

7. Wyss M, Kaddurah-Daouk R. Creatine and creatinine metabolism. Physiol Rev 2000; 80 (3): 1107-213.

8. Levey AS. Measurement of renal function in chronic renal disease. Kidney Int 1990; 38 (1): 167-84.

9. Simon J, Amde M, Poggio ED. Interpreting the estimated glomerular filtration rate in primary care: Benefits and pitfalls. Cleve Clin J Med 2011; 78 (3): 189-95.

10. Hottelart C, El Esper N, Rose F, Achard J-M, Fournier A. Fenofibrate increases creatininemia by increasing metabolic production of creatinine. Nephron 2002; 92 (3): 536-41.

11. Jaffe M. Ueber den Niederschlag welchen Pikrinsäure in normalen Harn erzeugt und über eine neue reaction des Kreatinins. Z Physiol Chem 1886; 10: 391-400.
12. Delanghe JR, Speeckaert MM. Creatinine determination according to Jaffe - What does it stand for? NDT Plus 2011; 4 (2): 83-6.

13. Panteghini M. Enzymatic assays for creatinine: Time for action. Clin Chem Lab Med 2008; 46 (4): 567-72.

14. Guder WG, Hoffmann GE, Hubbuch A, Poppe WA, Siedel J, Price CP. Multicentre evaluation of an enzymatic method for creatinine determination using a sensitive colour reagent. J Clin Chem Clin Biochem 1986; 24 (11): 889-902.

15. Crocker H, Shephard MD, White GH. Evaluation of an enzymatic method for determining creatinine in plasma. J Clin Pathol 1988; 41 (5): 576-81.

16. Myers GL, Miller WG, Coresh J, Fleming J, Greenberg $\mathrm{N}$, Greene T, et al. Recommendations for improving serum creatinine measurement: A report from the Laboratory Working Group of the National Kidney Disease Education Program. Clin Chem 2006; 52 (1): 5-18.

17. Piéroni L, Delanaye P, Boutten A, Bargnoux A-S, Rozet E, Delatour V, et al. A multicentric evaluation of IDMS-traceable creatinine enzymatic assays. Clin Chim Acta 2011; 412 (23-24): 2070-5.

18. Boutten A, Bargnoux A-S, Carlier M-C, Delanaye P, Rozet E, Delatour V, et al. Enzymatic but not compensated Jaffe methods reach the desirable specifications of NKDEP at normal levels of creatinine. Results of the French multicentric evaluation. Clin Chim Acta 2013; 419: 132-5.

19. Drion I, Cobbaert C, Groenier KH, Weykamp C, Bilo HJ, Wetzels JF, et al. Clinical evaluation of analytical variations in serum creatinine measurements: why laboratories should abandon Jaffe techniques. BMC Nephrol 2012; 13 (1): 133.

20. Kuster N, Cristol J-P, Cavalier E, Bargnoux A-S, Halimi $\mathrm{J}-\mathrm{M}$, Froissart M, et al. Enzymatic creatinine assays allow estimation of glomerular filtration rate in stages 1 and 2 chronic kidney disease using CKD-EPI equation. Clin Chim Acta 2014; 428: 89-95.

21. Lee E, Collier CP, White CA. Interlaboratory Variability in Plasma Creatinine Measurement and the Relation with Estimated Glomerular Filtration Rate and Chronic Kidney Disease Diagnosis. Clin J Am Soc Nephrol 2017; 12 (1): 29-37.

22. Herget-Rosenthal S, Kribben A, Pietruck F, Ross B, Philipp T. Two by two hour creatinine clearance-repeatable and valid. Clin Nephrol 1999; 51 (6): 348-54.

23. Cockcroft DW, Gault MH. Prediction of creatinine clearance from serum creatinine. Nephron 1976; 16 (1): 31-41.

24. Jelliffe RW. Creatinine clearance: bedside estimate. Ann Intern Med 1973; 79 (4): 604-5. 
25. Edwards K, Whyte H. Plasma creatinine level and creatinine clearance as tests of renal function. Australas Ann Med 1959; 8: 218-24.

26. Levey AS, Bosch JP, Lewis JB, Greene T, Rogers N, Roth D. A more accurate method to estimate glomerular filtration rate from serum creatinine: A new prediction equation. Ann Intern Med 1999; 130 (6): 461-70.

27. Levey AS, Coresh J, Greene T, Stevens LA, Zhang YL, Hendriksen S, et al. Using standardized serum creatinine values in the modification of diet in renal disease study equation for estimating glomerular filtration rate. Ann Intern Med 2006; 145 (4): 247-54.

28. Stevens LA, Coresh J, Feldman HI, Greene T, Lash JP, Nelson RG, et al. Evaluation of the modification of diet in renal disease study equation in a large diverse population. J Am Soc Nephrol 2007; 18 (10): 2749-57.

29. Levey AS, Stevens LA, Schmid CH, Zhang YL, Castro AF, Feldman HI, et al. A new equation to estimate glomerular filtration rate. Ann Intern Med 2009; 150 (9): 604-12.

30. Pottel H, Hoste L, Dubourg L, Ebert N, Schaeffner E, Eriksen BO, et al. An estimated glomerular filtration rate equation for the full age spectrum. Nephrol Dial Transplant 2016; 31 (5): 798-806.

31. Schaeffner ES, Ebert N, Delanaye P, Frei U, Gaedeke J, Jakob O, et al. Two novel equations to estimate kidney function in persons aged 70 years or older. Ann Intern Med 2012; 157 (7): 471-81. 